\title{
Endocrine dysfunction in psychology during social distancing measures
}

\author{
Javed $\mathrm{N}^{1}$, Khawaja $\mathrm{H}^{1}$, Malik $\mathrm{J}^{2}$, Ahmed $\mathrm{Z}^{2}$ \\ Shifa College of Medicine, Shifa Tameer-e-Millat University, Islamabad, Pakistan. nismatjaved@gmail.com
}

\begin{abstract}
OBJECTIVES: To determine the psychological impact of the social distancing measures in place due to the coronavirus pandemic.

METHODS: We conducted a cross-sectional study on the Pakistani population. Informed consent was taken from all the participants. The data was collected through an online questionnaire. Cronbach's alpha was used to assess the internal consistency of the questionnaire, and it was found to be 0.80 . The data obtained was analyzed on IBM's statistical package for the social sciences (SPSS) version 26.

RESULTS: Out of 706 participants, $489(69.26 \%)$ were males and $217(30.74 \%)$ were females. The mean age of the participants was $35.24 \pm 12.08$ years. The majority of the participants were from Punjab $(66.00 \%)$. The mean time since quarantine measures had been established was $10.35 \pm 5.09$ days. The mean total score was $9.08 \pm 2.38$ points. A majority of the participants $(25.64 \%)$ were daily wage workers. t-test was significant when the time of quarantine was compared to the psychological impact. Significant results were also found when gender was compared to the impact.

CONCLUSIONS: Social distancing measures have an impact on psychology and endocrinology of people in general. The impact can take the shape of long-lasting consequences (Tab. 3, Ref. 19). Text in PDF www.elis.sk KEY WORDS: quarantine, psychology, coronavirus, social distancing, Pakistan.
\end{abstract}

\section{Introduction}

The novel coronavirus was declared as public health emergency by the World Health Organization on 30 January 2020 (1, 2). The outbreak was first revealed in late December 2019, when clusters of pneumonia cases of unknown aetiology were found to be associated with epidemiologically linked exposure to a seafood market and untraced exposures in the city of Wuhan of Hubei Province (3).

Coronavirus is a beta-coronavirus that can spread to humans through intermediate hosts or through respiratory droplets (4). The average incubation period is 4 to 7 days (5). The spectrum of clinical features varies significantly from acute respiratory distress to severe cardiac injury (6). The case fatality rate ranges from $3 \%$ to $6 \%(7)$.

There is an element of fear among the public due to the ongoing pandemic. Social distancing measures are being employed to combat the pandemic. However, these measures are associated with significant psychological and mental consequences. There are few short-term effects such as: irritability, lack of freedom, and boredom from lack of social contact. Considering the previous pande-

${ }^{1}$ Shifa College of Medicine, Shifa Tameer-e-Millat University, Islamabad, Pakistan, and ${ }^{2}$ Rawalpindi Institute of Cardiology, Rawalpindi, Pakistan

Address for correspondence: $N$. Javed, Shifa College of Medicine, Shifa Tameer-e-Millat University NCBMS Tower, Near FBISE Office, Pitras Bukhari Road, Sector H-8/4, Islamabad, 44000, Pakistan mics, many people had post-traumatic stress disorder after quarantine measures were removed (8). There were also reports of anger issues and generalized anxiety disorders (9). In fact, the healthcare workers also considered resignation after the pandemic was resolved (10).

A few factors were thought to play a role, such as: occupation, preparedness, work environments, and social support (11). Currently, there is no evidence on the psychological impact of quarantine measures on the l public in Pakistan during the peak of the novel coronavirus epidemic. There are limited data on the physiologic mechanisms responsible for the consequences. Because of the alarming rates of spread in all the states of Pakistan, the lockdown was imposed from April. In this situation, daily wage workers were the most affected people prone to the psychosocial effects of quarantine. This is especially pertinent with the uncertainty surrounding an outbreak of such unparalleled magnitude.

Therefore, this present study represents the first psychological impact and mental health survey conducted in the general population in Pakistan within the first two weeks of quarantine for coronavirus outbreak. This study was aimed to establish the prevalence of psychiatric symptoms and identify risk and protective factors contributing to psychological stress as well as to report few mechanisms responsible for the psychological impact.

\section{Methods}

We conducted a cross-sectional study on people from all over Pakistan. The study was approved by the Institutional Review 
Board of Rawalpindi Institute of Cardiology, Rawalpindi (IRB \# 933-208-2019). The people, who were willing to participate in the study were included in the study. The people, who were not present during the days of the study were not included in the study. There were many measures employed to ensure the distribution of questionnaires. The questionnaires were distributed through online forms, on social media pages and in hard copies. Invitations to fill out the forms were also sent through emails.

The data were collected by the administration of a self-constructed questionnaire. It compromised two sections. The first section contained questions about the demographic information of the participants, while the second section contained questions assessing the impact of quarantine measures on the participants' routine. The second section was further divided into seven domains. The seven domains were as follows: the extent of work affected by quarantine measures, duration of sleep change in sleep pattern, change in eating habits, change in physical activity, the impact of work from home and depression. The domain for the extent of work was divided into six categories, which were scored from one to six points, with one point for the smallest impact and six points for severe impact. A similar scoring pattern was used for the duration of sleep, which was divided into four categories. The score ranged from one to four points with four points implying a severe reduction in sleep duration. The rest of the five domains were scored using a similar scheme. The 'yes' response was given one point in all these domains. Cronbach's alpha was used to assess the internal consistency of the questionnaire and was found to be 0.80 .

The scores in all the domains were then added. The highest possible total score was 15 . This score reflected the psychological impact of the quarantine measures. A total of 800 questionnaires were distributed, out of which 706 questionnaires (88.3\%) were filled. After the data collection, all the participants were given a seminar on the pandemic. A question and answer session was held after the seminar to address any queries regarding the novel coronavirus.

The data obtained were analysed on IBM's statistical package for the social sciences (SPSS) version 26 (IBM, Armonk, NY). Descriptive statistics were used to analyse and describe the data. Frequencies and percentages were calculated for qualitative variables like gender and occupation. The mean and standard deviation (SD) were calculated for quantitative variables such as age and domain scores. The Pearson chi-square test was applied to determine whether there was any significant difference between gender and the domain scores. The independent sample t-test was used to assess the time of quarantine with various variables. Pvalue of less than $<0.05$ was considered significant.

Tab. 1. Occupations of the participants.

\begin{tabular}{lc}
\hline Occupation & Frequency (\%) \\
\hline Daily wage workers & $181(25.64)$ \\
Business & $134(18.98)$ \\
Nurse & $109(15.43)$ \\
Student & $90(12.76)$ \\
Engineer & $76(10.76)$ \\
Doctor & $67(9.49)$ \\
Architect & $29(4.11)$ \\
Teacher & $20(2.83)$ \\
\hline
\end{tabular}

Tab. 2. Mean domain scores of the participants.

\begin{tabular}{lcc}
\hline Domain & Mean \pm SD & $\mathrm{p}$ \\
\hline Extent of work affected & $3.73 \pm 1.35$ & 0.00 \\
Duration of sleep & $2.01 \pm 0.90$ & 0.00 \\
Change in sleep pattern & $0.68 \pm 0.47$ & 0.00 \\
Change in eating habits & $0.80 \pm 0.40$ & 0.00 \\
Change in physical activity & $0.73 \pm 0.44$ & 0.00 \\
Depression & $0.31 \pm 0.26$ & 0.00 \\
Impact of 'work from home' & $0.83 \pm 0.37$ & 0.00 \\
\hline
\end{tabular}

$\mathrm{SD}=$ standard deviation

Tab. 3. Mean domain scores of both the genders.

\begin{tabular}{llcc}
\hline Domain & Gender & Mean \pm SD & $\mathrm{p}$ \\
\hline \multirow{2}{*}{ Extent of work affected } & Male & $4.23 \pm 1.00$ & \multirow{2}{*}{0.00} \\
\hline \multirow{2}{*}{ Duration of sleep } & Female & $2.59 \pm 1.34$ & \\
\hline \multirow{2}{*}{ Change in sleep pattern } & Male & $2.13 \pm 0.93$ & \multirow{2}{*}{0.00} \\
\hline \multirow{2}{*}{ Change in eating habits } & Male & $0.73 \pm 0.78$ & \\
\hline \multirow{2}{*}{ Change in physical activity } & Female & $0.53 \pm 0.50$ & \multirow{2}{*}{0.00} \\
\hline \multirow{2}{*}{ Depression } & Male & $0.82 \pm 0.39$ & \multirow{2}{*}{0.01} \\
\hline \multirow{2}{*}{ Impact of 'work from home' } & Male & $0.74 \pm 0.44$ & \\
\hline Psychological Impact & Female & $0.83 \pm 0.38$ & \multirow{2}{*}{0.00} \\
\hline
\end{tabular}

$\mathrm{SD}=$ standard deviation

\section{Results}

Out of 706 participants, 489 (69.26 \%) were males and 217 ( $30.74 \%$ ) were females. The mean age of the participants was 35.24 \pm 12.08 years. Most of the participants were from Punjab (66.00\%), Kashmir (13.51\%), and Khyber Pakhtunkhwa (10.80\%). The occupational details of the participants are shown in Table 1.

The mean time since quarantine measures had been established was $10.35 \pm 5.09$ days. The mean total score was $9.08 \pm 2.38$. t-test was applied to assess if the difference in each domain score and time since quarantine was significant. p-value of less than 0.05 was considered significant. The results are in Table 2.

The domain scores and gender were also compared. They were all found to be significant. The results are shown in Table 3.

\section{Discussion}

This is the first study in Pakistan that explores how quarantine measures impacted mental health. The mean age of the participants was similar to those from another study investigating the importance of online surveys, but our study had a male to female participant ratio of 70:30 whereas the 2020 study had a male to female participant ratio of 50:50 (12).

The study revealed that the male participants suffered a greater loss of work $(4.23 \pm 1.00, p=0.00)$ and were more likely to be affected by the quarantine as opposed to the female participants. There are few explanations for this difference. In Pakistan, there 


\section{8-880}

is already an ongoing gender gap in the available employment opportunities and the Pakistani workforce is mostly dominated by males (13). This difference of genders in the workforce is also attributable to the fact that the daily wage workers are most likely affected by the measures (14). Our study revealed that 181 participants were daily wage workers and they were all male. This also explains, why the male participants felt that 'work from home' had impacted them more.

The female participants had fewer hours of sleep compared to the male participants. This is partly indicative of anxiety and short-term fragmentation of sleep leads to an increased anxietylike behaviour. There is a link between anxiety, sleep disturbances, and the rise of hormonal levels, particularly in women (15). The higher the rise of progesterone level from the start to the end of the menstrual cycle, the higher the sleep disturbances and anxiety (16). Our findings confirmed this link because the female participants had the mean age of 27 years and were therefore, premenopausal. The release of reproductive hormones is cyclical in nature and therefore, the female participants did not believe that their sleep habits had changed $(0.53 \pm 0.50)$ owing to the quarantine situation. The hormonal changes also explain why female participants had a lower score for depression.

There are differences in responses of male participants in the same domains. There are few explanations for these differences. Psychological stress stimuli cause inhibition of the axis and results in low testosterone levels (17). These levels increase the risk of depression (18). in men, which is one of the findings of the study. Change in sleeping habits is one of the features of sleep disorders and might present even as long hours of sleep. The most likely factor is low testosterone concentration (19). These combined features of depression and low testosterone levels ultimately result in the changes in eating habits and physical activity.

There are few limitations to the study. The sample size is small, when studying various regions of the country. Different age groups have not been accounted for in the study completely. The study mentions changes in activities as a part of domain scores but does not investigate the increase or decrease in activities. The study did not evaluate the impact of endocrinology completely because it was beyond the scope of the study.

\section{Learning points:}

Social distancing measures have an adverse psychological impact.

These effects are related to the difference in endocrine physiology of both genders.

\section{Conclusion}

Social distancing measures have an indirect impact on the psychology of people around the world. These effects are a direct result of endocrinological dysfunctions. Such dysfunctions may have long-lasting consequences and therefore, should be addressed properly. There is a general need to improve the mental health of the population so that the consequences can be avoided.

\section{References}

1. Wang C, Horby PW, Hayden FG, Gao GF. A novel coronavirus outbreak of global health concern. Lancet 2020; 395 (10223): 470-473.

2. Cao Y, Cai K, Xiong L. Corona Virus Disease 2019: A new severe acute respiratory syndrome from Wuhan in China. Acta Virol 2020; 64:210-215.

3. Nishiura H, Jung SM, Linton NM et al. The Extent of Transmission of Novel Coronavirus in Wuhan, China, 2020. J Clin Med 2020; 9 (2).

4. Guo YR, Cao QD, Hong ZS et al. The origin, transmission and clinical therapies on coronavirus disease 2019 (COVID-19) outbreak - an update on the status. Mil Med Res 2020; 7 (1): 11.

5. Li Q, Guan X, Wu P et al. Early Transmission Dynamics in Wuhan, China, of Novel Coronavirus-Infected Pneumonia. N Engl J Med 2020; 382 (13): 1199-1207.

6. Zhao S, Musa SS, Lin Q et al. Estimating the Unreported Number of Novel Coronavirus (2019-nCoV) Cases in China in the First Half of January 2020: A Data-Driven Modelling Analysis of the Early Outbreak. J Clin Med 2020; 9 (2).

7. Jung SM, Akhmetzhanov AR, Hayashi $K$ et al. Real-Time Estimation of the Risk of Death from Novel Coronavirus (COVID-19) Infection: Inference Using Exported Cases. J Clin Med 2020; 9 (2): 523.

8. Hawryluck L, Gold WL, Robinson S, Pogorski S, Galea S, Styra R. SARS control and psychological effects of quarantine, Toronto, Canada. Emerg Infect Dis 2004; 10 (7): 1206-1212.

9. Jeong H, Yim HW, Song Y J et al. Mental health status of people isolated due to Middle East Respiratory Syndrome. Epidemiol Health 2016; 38: e2016048.

10. Bai Y, Lin CC, Lin CY, Chen JY, Chue CM, Chou P. Survey of stress reactions among health care workers involved with the SARS outbreak. Psychiatr Serv 2004; 55 (9): 1055-1057.

11. Brooks SK, Dunn R, Amlôt R, Rubin GJ, Greenberg N. A Systematic, Thematic Review of Social and Occupational Factors Associated with Psychological Outcomes in Healthcare Employees During an Infectious Disease Outbreak. J Occup Environ Med. 2018; 60 (3): 248-257.

12. Geldsetzer P. Use of Rapid Online Surveys to Assess People's Perceptions During Infectious Disease Outbreaks: A Cross-sectional Survey on COVID-19. J Med Internet Res 2020; 22 (4): e18790.

13. Sarwar A, Imran MK. Exploring Women's Multi-Level Career Prospects in Pakistan: Barriers, Interventions, and Outcomes. Front Psychol 2019; 10: 1376.

14. Gupta AG, Moyer CA, Stern DT. The economic impact of quarantine: SARS in Toronto as a case study. J Infect 2005; 50 (5): 386-393.

15. Grubac Z, Sutulovic N, Ademovic A et al. Short-term sleep fragmentation enhances anxiety-related behavior: The role of hormonal alterations. PLoS One 2019; 14 (7): e0218920.

16. Sharkey KM, Crawford SL, Kim S, Joffe H. Objective sleep interruption and reproductive hormone dynamics in the menstrual cycle. Sleep Med 2014; 15 (6): 688-693.

17. Nargund VH. Effects of psychological stress on male fertility. Nat Rev Urol 2015; 12 (7): 373-382.

18. McHenry J, Carrier N, Hull E, Kabbaj M. Sex differences in anxiety and depression: role of testosterone. Front Neuroendocrinol 2014; 35 (1): 42-57.

19. Wittert G. The relationship between sleep disorders and testosterone in men. Asian J Androl 2014; 16 (2): 262-265.

Received April 25, 2020. Accepted April 30, 2020. 